\title{
Effect of Bladder Distention Following Water Ingestion on Cardiac Autonomic Balance in Healthy Young Men
}

\author{
S. Mehran Hosseini ${ }^{1}$, Hamid Sepehri*1
}

1. Neuroscience Research Center, Department of Physiology, Faculty of Medicine, Golestan University of Medical Sciences, Gorgan, Iran

\begin{abstract}
Background and objectives: Bladder filling and severe urgency to urinate may affect the cardiovascular system through viscero-visceral reflexes. In the cardiovascular system, postmicturition syncope is a well-known and clinically important consequence of this phenomenon. Despite the importance of such reflexes, limited numbers of studies have investigated the in vivo effects of bladder filling on the heart. The purpose of this study was to determine the effects of bladder filling through water ingestion on heart rate and the autonomic nervous system in healthy young men.
\end{abstract}

Methods: This was a descriptive-analytical study performed on 20 healthy male volunteer students. Five minutes after voluntary bladder emptying, the electrodes were attached and heart rate was recorded in the sitting position in a controlled environment. After five minutes of adjustment, data collection was started at $1 \mathrm{KHz}$ sampling frequency. Then, the participants continued drinking water until developing severe urgency to urinate. Heart rate was recorded at the peak of urgency and the data were compared with baseline values. All data were expressed as mean \pm standard deviation. Statistical analyses of data were performed using SPSS 16 at significance level of 0.05.

Results: Mean age, weight and height of the participants were $22.3 \pm 3.466$ years, $75.55 \pm 15.5 \mathrm{~kg}$ and $175.8 \pm 11.28 \mathrm{~cm}$, respectively. Mean heart rate was $74 \pm 12.23$ after the initial urination, $68.55 \pm 10.53$ when feeling severe urgency to urinate and $66.3 \pm 10.87$ after the second urination. The heart rate differed significantly between the three tested conditions $(\mathrm{P}<0.05)$. There was no significant difference in other parameters of heart rate variation including SDNN, RMSSD, RRM, PNN50, absolute and relative (normalized) low frequency and high frequency and their ratio (LF/HF).

Conclusion: Bladder distension secondary to drinking water has no effect on the heart autonomic balance.

Keywords: Bladder distension, Heart, Autonomic nervous system

\section{Received: 2019/11/12}

Revised:2019/11/25

Published:2019/12/12

*Correspondence: Hamid Sepehri, Neuroscience Research Center, Department of Physiology, Faculty of Medicine, Golestan University of Medical Sciences, Gorgan, Iran

Tel: +98-1732453515

Email: hamsep49@yahoo.com 


\section{INTRODUCTION}

Bladder dilation affects cardiac functioning through the autonomic nervous system (ANS), which is also the most important factor in maintaining heart rate. Although factors such as heat and stretching also directly affect the heart rate, different stimuli by a variety of visceral neural reflexes (compressive, volumetric and chemical) affect the heart rate (1). Visceral sensory afferents indirectly influence the cardiac autonomic balance (CAB). During bladder filling, various impulses are sent through the sensory and visceral sensory receptors to the spinal cord and other parts of the central nervous system, causing void sensation and autonomic nerve reflexes, thus altering the activity of somatic nervous system and neuromuscular mechanisms of bladder emptying. The extent of this sensory and motor activity may affect other organs through the visceral-visceral reflexes. In the cardiovascular system, postmicturition syncope is a well-known and clinically important consequence of this phenomenon. Despite the importance of such reflexes, reports about the effect of bladder filling on the heart under normal conditions are limited and require evaluating distention of hollow visceral organs such as the stomach (3), small intestine (4), rectum (5) and gallbladder (6). Bladder volume changes also have a major impact on cardiovascular functioning (7-9). Studies on the direct impact of bladder distention on blood pressure and its relationship with RR intervals in humans have demonstrated that bladder distention can increase the baroreceptor reflex in people with primary hypertension, thereby increasing the probability of post-micturition syncope (10). In a study on healthy young people, bladder distention via drinking fluids increased systolic and diastolic blood pressure with no impact on the heart rate (7). Increased blood pressure following severe urinary retention has been also reported in other studies on healthy individuals. Moreover, urinary retention also increases blood pressure in middle-aged individuals with hypertension (8). Electrophysiological studies have shown that bladder distention increases the activity of efferent fibers of the pelvic and hypogastric nerves (9). The bladder sensory neurons located in the subepithelial network contain granular vesicles containing norepinephrine. Bladder stretches and stimulation of the efferent fibers increases sympathetic activity and decreases vagal tone that can affect the cardiovascular system (10). In a study on the effect of bladder distention on cardiovascular reflexes in dogs, it was found that bladder distention elevates blood pressure and stimulates carotid sinusoidal receptors, thus leading to bradycardia, hypotension and vasodilatation in the legs. In addition, concomitant distention and carotid receptor stimulation produced similar cardiovascular reflexes and hypotension, while the degree of vasodilatation in the legs decreased (12). Evaluation of heart rate variability (HRV) as the variation of interval between two consecutive heartbeats allows non-invasive in vivo assessment of the balance between the sympathetic and parasympathetic branches of the ANS. The objective of this study was to determine the effects of bladder filling by drinking water on activity of the cardiac ANS in healthy individuals based on HRV indices.

\section{MATERIALS AND METHODS}

The study was performed on 20 eligible healthy male volunteer students (age range: 19-24 years) from the Golestan University of Medical Sciences, Iran. The participants had no history of disease, medication, smoking and physical activity. The subjects were enrolled in the study after fully explaining the research objectives and written consent was obtained from all participants. This clinical trial was approved by the Ethics Committee of the Golestan University of Medical Sciences, Iran. All tests were performed in the morning, in a silent room and at $22 \pm 2{ }^{\circ} \mathrm{C}$. Following voluntary bladder emptying, electrodes of were attached and heart rate was monitored in sitting position under a controlled environment. After five minutes of 
Journal of Clinical and Basic Research (JCBR). 2019; 3(4): P 1-6.

adjustment to the test conditions, blood pressure and ECG were recorded for five minutes, with a sampling frequency of $1 \mathrm{kHz}$ to convert analog data to digital. Subsequently, the volunteers continued to drink water until developing a severe urgency to urinate. Then, heart's electrical activity and blood pressure were recorded once again. Oscillometric blood pressure was measured from the brachial artery and in the sitting position using a blood pressure monitor (OMRON, made in China). The heart's electrical activity was recorded continuously in the sitting position using electrodes of an ADinstrument device (Powerlab).

\section{Statistical analyses}

After performing the necessary calculations in the Chart 5 software, the collected data were expressed as mean value and standard deviation. All statistical analyses were performed in SPSS 16 using paired t-test and at significance level of 0.05 .

\section{RESULTS}

Table 1 presents the mean height, age and weight of the study subjects.

Table 1. Mean height, age and weight of the study subjects

\begin{tabular}{|c|c|c|c|}
\hline Variable & Age (year) & Weight $(\mathrm{kg})$ & Height $(\mathrm{cm})$ \\
\hline Mean & 22.30 & 75.5500 & 175.8000 \\
Standard deviation & 3.466 & 15.50373 & 11.28856 \\
Minimum & 18 & 48.00 & 147.00 \\
Maximum & 30 & 100.00 & 190.00 \\
\hline
\end{tabular}

Mean amount of water intake and urine volume were $1971 \pm 651.5 \mathrm{ml}$ (range: 1080 to $2880 \mathrm{ml}$ ) and $455 \pm 177.6 \mathrm{ml}$ (range: 100 to $750 \mathrm{ml}$ ), respectively. The time interval between water intake and urination was $1.38 \pm 0.19$ hours (range: 1 to 1.5 hours). Mean heart rate was $74 \pm 12.23$ after the initial urination, $68.55 \pm 10.53$ when feeling severe urgency to urinate and $66.3 \pm 10.87$ after the second urination. The heart rate differed significantly between the three tested conditions. There was no significant difference in other parameters of HRV over time. Tables 2, 3 and 4 present the mean values of the studied parameters at the three test conditions.

Table 2. Mean value of time interval indicators and HRV frequencies at baseline

\begin{tabular}{|c|c|c|c|c|c|c|c|}
\hline Parameter & LF $\left(\mathbf{m s}^{2}\right)$ & HF $\left(\mathbf{m s}^{2}\right)$ & LF/HF & SDNN (ms) & RMSSD & RRM (ms) & PNN50 (\%) \\
\hline $\begin{array}{c}\text { Mean value } \\
\begin{array}{c}\text { Standard } \\
\text { deviation }\end{array}\end{array}$ & 26934.7518 & 37426.1782 & .7855 & 83.3485 & 9.9400 & 1151.2000 & 21.3500 \\
& 13656.15969 & 20074.48099 & .34772 & 59.50146 & 6.54092 & 801.97287 & 16.90305 \\
\hline
\end{tabular}

LF: low frequency; HF: high frequency; LF/HF: LF to HF ratio; RMSSD: the square root of the mean of the squares of the successive differences between adjacent NNs; RRM: RR intervals mean; PNN50: the proportion of NN50 divided by total number of NNs.

Table 3. Mean value of time interval indicators and HRV frequencies when feeling the urgency to urinate after drinking water

\begin{tabular}{|c|c|c|c|c|c|c|c|}
\hline Parameter & IF2 $\left(\mathbf{m s}^{2}\right)$ & HF2 $\left(\mathbf{m s}^{2}\right)$ & IF/HF2 & SDNN2 (ms) & RMSSD2 & RRM2 (ms) & PNN502 (\%) \\
\hline Mean value & 29126.5245 & 43456.8817 & .7255 & 107.7227 & 11.7700 & 893.5500 & 27.4000 \\
Standard & 12425.65831 & 22284.58855 & .20874 & 90.65389 & 9.58069 & 127.80968 & 16.67459 \\
deviation & & &
\end{tabular}


LF: low frequency; HF: high frequency; LF/HF: LF to HF ratio; RMSSD: the square root of the mean of the squares of the successive differences between adjacent NNs; RRM: RR intervals mean; PNN50: the proportion of NN50 divided by total number of NNs.

Table 4. Mean value of time interval indicators and HRV frequencies after complete bladder emptying

\begin{tabular}{|c|c|c|c|c|c|c|c|}
\hline Parameter & IF3 $\left.\mathbf{( m s}^{\mathbf{2}}\right)$ & HF3 $\left(\mathbf{m s}^{\mathbf{2}}\right)$ & IF/HF3 & SDNN3 (ms) & RMSSD3 & RRM3 (ms) & PNN503 (\%) \\
\hline $\begin{array}{c}\text { Mean value } \\
\begin{array}{c}\text { Standard } \\
\text { deviation }\end{array}\end{array}$ & 26995.5628 & 44038.4190 & .6460 & 84.7976 & 8.9050 & 927.7500 & 30.3000 \\
& 1150.57715 & 25031.82357 & .18503 & 44.18157 & 4.27138 & 142.73600 & 16.60089 \\
\hline
\end{tabular}

LF: low frequency; HF: high frequency; LF/HF: LF to HF ratio; RMSSD: the square root of the mean of the squares of the successive differences between adjacent NNs; RRM: RR intervals mean; PNN50: the proportion of $\mathrm{NN} 50$ divided by total number of NNs.

\section{DISCUSSION}

Bladder filling and a severe urinary urgency may affect the cardiovascular system through autonomic reflexes. We utilized HRV as a valuable tool for assessing the ANS variation in response to bladder distention. As mentioned, the heart rate decreased significantly after bladder distention and restored to baseline value after the bladder was emptied. This finding is in line with findings of a study by Yamaguchi et al. that showed excessive bladder distention increases vagal tone and reduces heart rate via vagovagal reflexes (13). Similarly, Daly et al. reported that increased pressure and stimulation of carotid sinusoidal receptors leads to bradycardia and hypotension and concluded that reducing the activity of parasympathetic afferents can decrease heart rate during bladder distention (14). Inconsistent with these findings, Hassan et al. reported that bladder distention by hot water ingestion in anesthetized dogs can increase heart rate by decreasing the parasympathetic activity and increasing the sympathetic activity (15).

In our study, despite the increase in heart rate, we observed no significant difference in parameters of HRV over time and frequency range including low frequency (LF), high frequency (HF) and their ratio (LF/HF). However, a study claimed that bladder distention could change HRV variables in anesthetized individuals (16). Furthermore, bladder distention has been thought to stimulate vagovagal reflexes, thereby decreasing HF activity (13). There are also reports of sympathetic activation and increase in Lf via stimulation of viscero-vascular reflexes (17).

Guttmann et al. (1947) studied the effect of bladder distention on cardiovascular functioning in a group of World War II patients with spinal cord injury. Surprisingly, they found that bladder distention could raise blood pressure while there was no neural pathway to transmit impulses from the bladder to the brain. They suggested that accessory neural pathways within the viscera may transmit impulses to areas above the damaged spinal cord, thus inducing bladdercardiovascular reflexes (18). On the other hand, it has been observed that bladder distention can increase the level of circulating catecholamines (19). Since bladder distention can induce cardiovascular reflexes, it is essential to gain a better understanding of the causes of HRV consistency in our study by evaluating hormonal responses including variation of angiotensin.

\section{CONCLUSION}

Bladder distention by drinking water does not affect the CAB. Other mechanisms such as change in the blood pressure and hormones might compensate for the autonomic response.

\section{ACKNOWLEDGMENTS}

We would like to thank the Deputy of Research and Technology of Golestan 
University of Medical Sciences for the financial support and the Neuroscience Research Center of the University for cooperating in the experimentations.

\section{DECLARATIONS \\ Funding}

This study was supported by deputy of research and technology of Golestan University of Medical Sciences.

\section{Ethics approvals and consent to participate:}

The study was approved by the ethics committee of the Golestan University of Medical Sciences, Iran. A written informed consent was taken from all participants prior to participation.

\section{Conflict of interest}

The authors declare no conflict of interest.

\section{REFERENCES}

1. Kc P, Dick TE. Modulation of cardiorespiratory function mediated by the paraventricular nucleus. Respir Physiol Neurobiol. 2010;174(1-2):55-64. [DOI:10.1016/j.resp.2010.08.001]

2. Guzzetti S, Borroni E, Garbelli PE, Ceriani E, Della Bella P, Montano N, et al. Symbolic dynamics of heart rate variability: a probe to investigate cardiac autonomic modulation. Circulation. 2005;112(4):465-70. [DOI:10.1161/CIRCULATIONAHA.104.518449]

3. Longhurst JC, Stebbins CL, Ordway GA. Chemically induced cardiovascular reflexes arising from the stomach of the cat. Am J Physiol. 1984;247(3):H459-466.

[DOI:10.1152/ajpheart.1984.247.3.H459]

4. Cevese A, Mary DA, Poltronieri R, Schena F, Vacca G. Haemodynamic effects of distension of the descending colon in anaesthetized dogs. The Journal of physiology. 1992;447(1):409-23. [DOI:10.1113/jphysiol.1992.sp019009]

5. Ness TJ, Gebhart GF. Characterization of neurons responsive to noxious colorectal distension in the T13-L2 spinal cord of the rat. $\mathbf{J}$ Neurophysiol. 1988;60(4):1419-38. [DOI:10.1152/jn.1988.60.4.1419]
6. Martin SE, Pilkington DM, Longhurst JC. Coronary vascular responses to chemical stimulation of abdominal visceral organs. Am J Physiol. 1989;256(3):H735-744.

[DOI:10.1152/ajpheart.1989.256.3.H735]

7. Szasz JJ, Whyte HM. Effect of distension of the bladder and of contraction of sphincters on blood pressure. $\mathrm{Br}$ Med J. 1967;2(5546):208-10. [DOI:10.1136/bmj.2.5546.208]

8. Lee T-M, Su S-F, Chen M-F, Tsai C-H. Acute effects of urinary bladder distention on the coronary circulation in patients with early atherosclerosis. J Am Coll Cardiol. 2000;36(2):453-60.

1097(00)00751-8]

[DOI:10.1016/S0735-

9. Floyd K, Hick VE, Morrison JF. Mechanosensitive afferent units in the hypogastric nerve of the cat. The Journal of physiology. 1976;259(2):457-71.

[DOI:10.1113/jphysiol.1976.sp011476]

10. Dixon JS, Gilpin CJ. Presumptive sensory axons of the human urinary bladder: a fine structural study. Journal of anatomy. 1987;151:199.

11. Giuliani S, Maggi CA, Meli A. Capsaicinsensitive afferents in the rat urinary bladder activate a spinal sympathetic cardiovascular reflex. Naunyn Schmiedebergs Arch Pharmacol. 1988;338(4):411-6. [DOI:10.1007/BF00172119]

12. de Burgh Daly M, Ward J, Wood LM. Effects of distension of the urinary bladder on the cardiovascular reflexes from the carotid baroreceptors in the dog. J Physiol. 1993;463:54564. [DOI:10.1113/jphysiol.1993.sp019610]

13. Yamaguchi Y, Tsuchiya M, Akiba T, Yasuda M, Kiryu Y, Hagiwara $T$, et al. Nervous Influences Upon The Heart Due To Overdistension Of The Urinary Bladder: The Relation Of Its Mechanism To Vago-Vagal Reflex. Keio J Med. 1964;13:87-99. [DOI:10.2302/kjm.13.87]

14. de Burgh Daly M, Wood LM, Ward J. Cardiovascular responses to carotid chemoreceptor stimulation in the dog: their modulation by urinary bladder distension. J 
Journal of Clinical and Basic Research (JCBR). 2019; 3(4): P 1-6.

Physiol. 2000;524 Pt 3(Pt 3):903-917.

[DOI:10.1111/j.1469-7793.2000.00903.x]

15. 15 Hassan AA, Hicks MN, Walters GE, Mary DA. Effect on efferent cardiac vagal nerve fibres of distension of the urinary bladder in the dog. Q J Exp Physiol Camb Engl. 1987;72(4):473-81. [DOI:10.1113/expphysiol.1987.sp003089]

16. Prakash S, Kale S, Mullick P, Gogia AR. Cardiovascular manifestations of perioperative acute urinary bladder over-distension. Indian $\mathbf{J}$ Anaesth. 2014;58(4):502. [DOI:10.4103/00195049.139033]

17. Fagius J, Karhuvaara S. Sympathetic activity and blood pressure increases with bladder distension in humans. Hypertens. 1989;14(5):5117. [DOI:10.1161/01.HYP.14.5.511]

18. Guttmann L, Whitteridge D. Effects of bladder distension on autonomic mechanisms after spinal cord injuries. Brain J Neurol. 1947;70(4):361-404. [DOI:10.1093/brain/70.4.361]

19. Frankel HL, Mathias CJ, Walsh JJ. Blood pressure, plasma catecholamines and prostaglandins during artificial erection in a male tetraplegic. Paraplegia. 1974;12(3):205-1 [DOI:10.1038/sc.1974.33] 